\title{
Effective Survey on Detection and Classification of COVID-19 Suspected Individual Using CT scan Images
}

\author{
Snehal R. Sambhe ${ }^{1}$, Dr. Kamlesh A. Waghmare ${ }^{2}$
}

${ }^{1}$ Department of Computer Science and Engineering, Government College of Engineering, Amravati, Maharashtra, India

${ }^{2}$ Assistant Professor, Department of Computer Science and Engineering, Government College of Engineering, Amravati, Maharashtra, India

Article Info

Volume 7, Issue 3

Page Number: 294-299

Publication Issue :

May-June-2021

\section{Article History}

Accepted : 15 May 2021

Published : 22 May 2021

\section{ABSTRACT}

As insufficient testing kits are available, the development of new testing kits for detecting COVID remains an open vicinity of research. It's impossible to test each and every patient suffering from coronavirus symptoms using the traditional method i.e. RT-PCR. This test requires more time to produce results and have less sensitivity. Detecting feasible coronavirus infection using chest $\mathrm{X}$ Ray may also assist quarantine excessive risk sufferers while testing results are disclosed. A learning model can be built based on CT scan images or Chest Xrays of individuals with higher accuracy. This paper represents a computer-aided diagnosis of COVID 19 infection bases on a feature extractor by using CNN models.

Keywords: COVID 19, Classification, Corona virus, Chest Computed Tomography images

\section{INTRODUCTION}

The outbreak of COVID-19, which is additionally referred to as coronavirus. Pneumonia along with cough, fatigue, fever, muscle pain are the symptoms of this virus. In critical condition, the person may suffer from shortness of breath and severe headaches too. The very first patient who was affected by coronavirus has been found in December 2019 in Wuhan, a town in China. The diseases get spread mainly via respiratory droplets of coronavirusinfected patients. The novel coronavirus can also be spread if an unaffected person comes in contact with the droplets of an infected person and then touches their own face specially nose, eyes, or mouth without cleaning hands. Almost 210 countries get infected by this novel coronavirus, declared by WHO i.e. World Health Organization on April 25, 2020 [1]. It's impractical to have medicine or vaccine for COVID19 sufferers as the reason for pneumonia and the potential to a create new strain is unknown. So, in excessive alert region number of tests must be increased and physical distancing is began out in exercise amongst human beings.

RT-PCR or real-time polymerase chain reaction has been followed as well known diagnostic approach for 
the detection of coronavirus contamination in COVID-19 sufferers. This test requires $4-5$ hours or maybe an entire day to provide the end results. As the duration of producing the result of the test is greater than the speed of spreading coronavirus among human beings, detection using CT scan images of an infected person can be a solution. As compared to coronavirus inflamed people, the number of RT-PCR tests and the scarcity of testing kits makes RT-PCR tests useless. In contrast, CT scan and X-Ray technologies are broadly accepted conventional way of diagnosing people for several illnesses is a regular practice followed by radiologists for a couple of decades. It's difficult to supply enough number of testing kits in vastly affected zones. Thus, to deal with these issues, identification of COVID can be done using chest CT images of the persons those are affected by the symptoms of COVID-19.

The COVID-19 contamination is growing at a fast rate, with fewer number of kits available. Therefore, the improvement in the availability of testing kits remains an open vicinity of research. Newly,researches have proven that CT scan images may be used for testing COVID-19, as they display symmetric changes in COVID inflamed person. The testing of COVID sufferers based on their computed tomography images isn't a smooth venture as it predicts symmetric changes. Automatic classification of COVID inflamed person based on their CT images is the primary goal of this paper [2]. As the computed tomography scanners are available in most of the hospitals, classification based on this can be implemented under the observation of expert physicians with greater speed. Thus, classification and detection of COVID-19 sufferers can be implemented using a supervised machine learning model based on chest images

\section{RELATED WORK}

There is a lot of development in medical as well as image-processing techniques, which helps in increasing the tools required for diagnosis and predicting the illnesses [3]. Different ML (Machine Learning) approaches are commonly used as a wellknown tool to enhance the diagnosis of many illnesses [4,5]. Better ML models are obtained and implemented using prominent feature extractors. That's why DL are widely used and accepted in a clinical imaging system as they automatically extract important features or by applying some predefined neural networks like a residual network [6]. The accuracy of proposed the model in imageCLEF2015 is $76.87 \%$ and in imageCLEF2016 is $87.37 \%$. Nardelli P. et al. [7] used 3D-CNN for the classification of pulmonary artery veins from CT-scan images and achieves an accuracy of $94 \%$.

Shin H. et al. [8] implemented a deep-CNN model for classifying lung illnesses in CT-scan images. This research explored and evaluated three different architectures of CNN like GoogleNet AlexNet, CifarNet with different training parameters. A Malignant lung nodules classification using collaborative DL was introduced by Xie Y. et al. With 95.70\% accuracy [9]. Hagerty J. et al. [10] categorized the Melanoma-dermoscopy-images by combining convolutional image processing with Deep-learning and gives classification accuracy of 0.94 . Gerard S. et al. [11] discovered a discriminative learning model in which detection of the fissure was evaluated with methods like U-Net, Fissure-Net, Hessian, and DoS. Setio A. et al. [12] implemented multi view ConvNet to identify the pulmonary hard nodules in CT-scan images and obtained an accuracy of 0.996. In this, features from CT images are automatically learned from training data. Xia K. et al. [13] generated a deep adversarial based model with the combination of loss 
functions for performing segmentation on abdominal images.

Zreik M. et al. [14] used R-CNN i.e. recurrent-CNN to classify stenosis and plaque in coronary scan images. This method achieves $77 \%$ accuracy for characterization and detection of plaque and $80 \%$ for stenosis characterization and detection. Bhandary A. et al. [15] presented a technique for lung irregularity detection using neural learning and achieved 97.27\% accuracy. Gao X et al. [16] used a 3D-block-based residual neural network for predicting the severity of TB i.e. tuberculosis in pulmonary images of respective sufferers. Pannu et al. $[17,18]$ implemented a model for Swarm particle optimization using the Neuro Fuzzy system, as the rate of classification can be increased. Zeng X. et al. [19] designed GCNN i.e Grated bidirectional CNN to identify infected suffers, as it is established that DL-based systems give remarkable results. The results of this technique can be further improved using variations in the residual network, which is an efficient strategy for feature extraction [20]. Transfer learning can be used for hyper tuning the learning models. Nabin et al. [21] used image-processing techniques to obtain important features like narrow vessels, lesion distribution patterns, atypical pigment network, and machine learning techniques to detect melanoma in Dermoscopy Images. Pathan S. et al. [22] has given a review on algorithms and different techniques for diagnosing pigmented skin.

You J. et al. [23] implemented a neural network using a linear classifier to predict drug-target interactions (DTIs), along with the advantage of re-purposing drugs for patients having breast cancer. In this model LASSO i.e Least Absolute Shrinkage and Selection Operator models for classification and LASSO DeepNN model for feature selection is used to identify DTIs. Lo et al. [24] used Convolutional-NN specially for the training purpose of the pulmonary nodule and implemented on radiography images. Yue et al. [25] predicted the drug-drug interactions (DDIs) model. Imbalanced data and selection of features were also detected. In order to enhance the accuracy of the DDIs prediction model, a multilayered feature selector and oversampling model were used.

\section{APPROCH REGARDING PROPOSED METHODOLOGY}

The aim of using CNN is to obtain greater accuracy for classification purposes by separating the positives sufferer's chest-radiography images from the negative sufferer's chest-radiography images. The powerful ConvNet makes use of various filters for extracting important features from the image by preserving the position data of pixels. The images are represented in the form matrix which consists of numbers or pixels and a mathematical operation i.e Convolution is performed on this matrix. Convolution captures spatial features of the image.

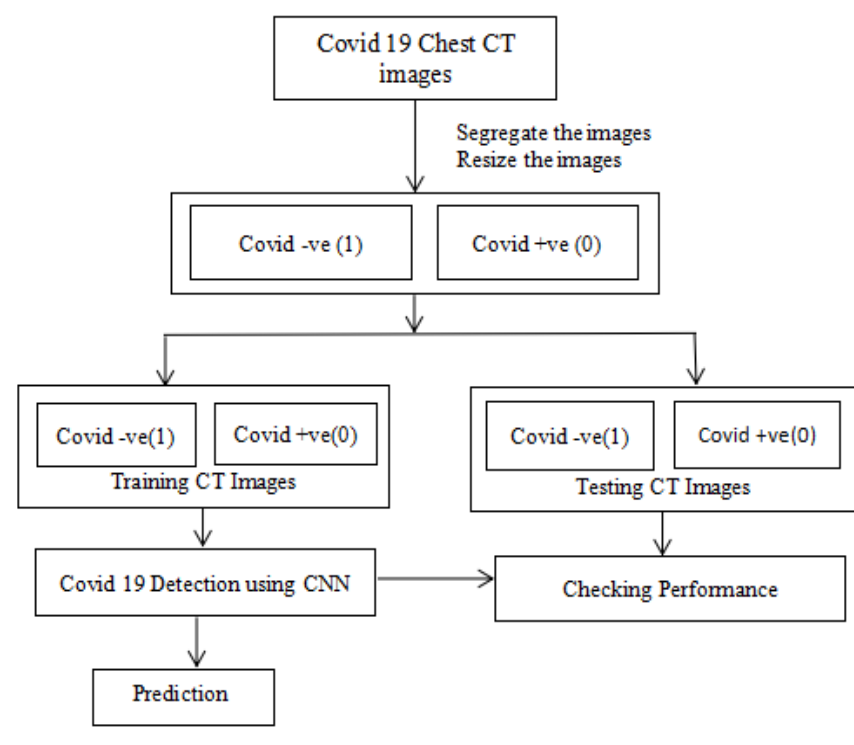

Figure 1. Proposed Methodology 


\section{CONCLUSION}

The current situation of COVID-19 has been announced as a global health emergency because of the quite excessive contamination rate of the illness. No proper drug/vaccine for curing COVID-19 sufferers is available at this moment. Early detection of virus-affected individuals is essential for interrupting inter-human transmission. In order to prevent the spread of this pandemic, the only solution we have is to quarantine or isolate the sufferers. Today, CT images of virus victims having some symptoms/signs are the effective way for identifying COVID-19. This paper presents a DLbased feature extractor and classifier approach to make a diagnosis of this illness. A well-known ConvNet architecture is used for extracting crucial features and identifying victims based on their respective CAT scans. And the formal Deep feature extraction is taken into consideration as a crucial step while implementing Deep-NN models.

\section{REFERENCES}

[1]. Kabid Hassan Shibly, Samrat Kumar Dey, Md Tahzib-Ul Islam, Md Mahbubur Rahman, COVID faster R-CNN: A novel framework to Diagnose Novel Coronavirus Disease (COVID19) in X-Ray images, Informatics in Medicine Unlocked, Volume 20, 2020, 100405, ISSN 2352-9148,

https://doi.org/10.1016/j.imu.2020.100405.

[2]. Ezz El-Din Hemdan and Marwa A. Shouman and Mohamed Esmail Karar. COVIDX-Net: A Framework of Deep Learning Classifiers to Diagnose COVID-19 in X-Ray Images. 2020, eprint:2003.11055.

[3]. Kaur, M., Singh, D. Fusion of medical images using deep belief networks. Cluster Comput 23, 1439-1453

(2020). https://doi.org/10.1007/s10586-019-02999-x.
[4]. Kumar Shukla P, Kumar Shukla P, Sharma P, Rawat P, Samar J, Moriwal R, Kaur M. Efficient prediction of drug-drug interaction using deep learning models. IET Syst Biol. 2020 Aug;14(4):211-216. doi: 10.1049/ietsyb.2019.0116. PMID: 32737279.

[5]. Manjit Kaur,Hemant Kumar Gianey, Dilbag Singh, Munish Sabharwal. Multi-objective differential evolution based random forest for e-health applications, Journal Article 2019, Modern Physics Letters B, 1950022, 33(05), doi1: 0.1142/S0217984919500222

[6]. Yu Y., Lin H., Meng J., Wei X., Guo H., Zhao Z.; Deep Transfer Learning for Modality Classification of Medical Images. Information 2017, 8, 91. https://doi.org/10.3390/info8030091

[7]. Nardelli, P., Jimenez-Carretero, D., BermejoPelaez, D., Washko, G. R., Rahaghi, F. N., Ledesma-Carbayo, M. J., \& San Jose Estepar, R. (2018). Pulmonary Artery-Vein Classification in CT Images Using Deep Learning. IEEE transactions on medical imaging, 37(11), 24282440 .

https://doi.org/10.1109/TMI.2018.2833385

[8]. Shin, H. C., Roth, H. R., Gao, M., Lu, L., Xu, Z., Nogues, I., Yao, J., Mollura, D., \& Summers, R. M. (2016). Deep Convolutional Neural Networks for Computer-Aided Detection: CNN Architectures, Dataset Characteristics and Transfer Learning. IEEE transactions on medical imaging, 35(5), 1285-1298. https://doi.org/10.1109/TMI.2016.2528162

[9]. Xie, Y., Xia, Y., Zhang, J., Song, Y., Feng, D., Fulham, M., \& Cai, W. (2019). Knowledgebased Collaborative Deep Learning for BenignMalignant Lung Nodule Classification on Chest CT. IEEE transactions on medical imaging, $38(4)$, 991-1004. https://doi.org/10.1109/TMI.2018.2876510

[10]. Hagerty, J. R., Stanley, R. J., Almubarak, H. A., Lama, N., Kasmi, R., Guo, P., Drugge, R. J., 
Rabinovitz, H. S., Oliviero, M., \& Stoecker, W. V. (2019). Deep Learning and Handcrafted Method Fusion: Higher Diagnostic Accuracy for Melanoma Dermoscopy Images. IEEE journal of biomedical and health informatics, 23(4), $1385-1391$. https://doi.org/10.1109/JBHI.2019.2891049

[11]. Gerard, S. E., Patton, T. J., Christensen, G. E., Bayouth, J. E., \& Reinhardt, J. M. (2019). FissureNet: A Deep Learning Approach For Pulmonary Fissure Detection in CT Images. IEEE transactions on medical imaging, 38(1), 156-166.

https://doi.org/10.1109/TMI.2018.2858202

[12]. Setio, A. A., Ciompi, F., Litjens, G., Gerke, P., Jacobs, C., van Riel, S. J., Wille, M. M., Naqibullah, M., Sanchez, C. I., \& van Ginneken, B. (2016). Pulmonary Nodule Detection in CT Images: False Positive Reduction Using Multi-View Convolutional Networks. IEEE transactions on medical imaging, 35(5), 1160-1169. https://doi.org/10.1109/TMI.2016.2536809

[13]. XIA, Kaijian \& YIN, Hongsheng \& Qian, Pengjiang \& Jiang, Yizhang \& Wang, Shuihua. (2019). Liver Semantic Segmentation Algorithm Based on Improved Deep Adversarial Networks in Combination of Weighted Loss Function on Abdominal CT Images. IEEE Access. PP. 1-1. 10.1109/ACCESS.2019.2929270.

[14]. Zreik, M., van Hamersvelt, R. W., Wolterink, J. M., Leiner, T., Viergever, M. A., \& Isgum, I. (2019). A Recurrent CNN for Automatic Detection and Classification of Coronary Artery Plaque and Stenosis in Coronary CT Angiography. IEEE transactions on medical imaging, 38(7), 1588-1598. https://doi.org/10.1109/TMI.2018.2883807

[15]. Bhandary, Abhir \& Prabhu, Ananth \& Rajinikanth, Venkatesan \& Krishnan, Palani \& Satapathy, Suresh \& Robbins, David \& Shasky,
Charles \& Zhang, Yu-Dong \& Tavares, Joao \& Raja, N. (2020). Deep-learning framework to detect lung abnormality - A study with chest X-Ray and lung CT scan images. Pattern Recognition Letters. $129 . \quad 271-278$. 10.1016/j.patrec.2019.11.013.

[16]. Xiaohong W. Gao, Carl James-Reynolds, Edward Currie. Analysis of tuberculosis severity levels from CT pulmonary images based on enhanced residual deep learning architecture, Neurocomputing, Volume 392, 2020, Pages 233-244, ISSN 0925-2312, https://doi.org/10.1016/j.neucom.2018.12.086.

[17]. Pannu, H.S., Singh, D. and Malhi, A.K. (2018), Improved Particle Swarm Optimization Based Adaptive Neuro-Fuzzy Inference System for Benzene Detection. Clean - Soil, Air, Water, 46:

1700162.

https://doi.org/10.1002/clen.201700162.

[18]. Pannu, H.S., Singh, D. \& Malhi, A.K. Multiobjective particle swarm optimization-based adaptive neuro-fuzzy inference system for benzene monitoring. Neural Comput \& Applic 31, 2195-2205 (2019). https://doi.org/10.1007/s00521-017-3181-7

[19]. Xingyu Zeng, Wanli Ouyang, Bin Yang, Junjie Yan, Xiaogang Wang. Gated Bi-directional CNN for Object Detection. 2016, 14th European Conference on Computer Vision, ECCV.

[20]. Saha, Sujay \& Khabir, Kanij \& Abir, Shadman \& Islam, Ariful. (2019). A newly proposed object detection method using Faster R-CNN inception with ResNet based on Tensorflow. 34. 10.1117/12.2523930.

[21]. Nabin K. Mishra and M. Emre Celebi. An Overview of Melanoma Detection in Dermoscopy Images Using Image Processing and Machine Learning, 1601.07843, 2016.

[22]. Pathan, Sameena \& P C, Siddalingaswamy \& Prabhu, K. (2017). Techniques and algorithms 
for computer aided diagnosis of pigmented skin lesions-A review. Biomedical Signal Processing and Control. 39. 237-262. 10.1016/j.bspc.2017.07.010.

[23]. You, J., McLeod, R. D., \& Hu, P. (2019). Predicting drug-target interaction network using deep learning model. Computational biology and chemistry, 80, 90101.https://doi.org/10.1016/j.compbiolchem.201 9.03.016

[24]. Shih-Chung B. Lo, Heang-Ping Chan, JyhShyan Lin, Huai Li, Matthew T. Freedman, Seong K. Mun. Artificial convolution neural network for medical image pattern recognition, Neural Networks, Volume 8, Issues 7-8, 1995, Pages 1201-1214, ISSN 0893-6080, https://doi.org/10.1016/0893-6080(95)00061-5.

[25]. Yue, K., Zou, B., Wang, L., Li, X., Zeng, M. and Wei, F. (2017), Prediction of Drug-Drug Interactions Based on Multi-layer Feature Selection and Data Balance. Chinese J. Electron., $\quad$ 26: 585-590. https://doi.org/10.1049/cje.2017.04.005

\section{Cite this Article}

Snehal R. Sambhe, Dr. Kamlesh A. Waghmare, "Effective Survey on Detection and Classification of COVID-19 Suspected Individual Using CT scan Images", International Journal of Scientific Research in Computer Science, Engineering and Information Technology (IJSRCSEIT), ISSN : 2456-3307, Volume 7 Issue 3, pp. 294-299, May-June 2021. Available at doi : https://doi.org/10.32628/CSEIT217339 Journal URL : https://ijsrcseit.com/CSEIT217339 